6 patients did not attend for nurse review. 3 (50\%) did not require referral if the iLFT protocol had been followed.

The median number of times a patient had abnormal LFTs before referral was 5 ( $\min 1 ; \max 33)$. The median duration of abnormal LFTs before referral was 20 months (min 0; max $87)$, as shown in figure 1 .

Conclusions iLFTs rapidly and safely identify patients with abnormal LFTs requiring referral to secondary care. If iLFTs were used by referrers, $24.7 \%$ of patients would not require referral to secondary care. This represents a substantial cost saving in terms of clinic appointments and unnecessary investigations. In addition, patients would be analysed at the time of the first abnormal LFTs, providing referrers information on how to monitor for disease progression and when to refer. This would save significant time in Primary Care, avoiding repeated venepuncture and ensuring patients are referred before they develop end stage liver disease.

\section{PTU-023 INVESTIGATING LIVER FUNCTION IN THE DIABETIC POPULATION OF A DISTRICT GENERAL HOSPITAL}

S Mankodi*, P Patel, J Gertner, D Nissan, Sophie McKenna, D Suri. Whittington NHS Trust, London, UK

\subsection{6/gutjnl-2019-BSGAbstracts.232}

Background The rising prevalence of type 2 diabetes (T2DM) and its complications including non-alcoholic fatty liver disease (NAFLD) is now a leading cause of cirrhosis and hepatocellular cancer. Prompt identification of fibrosis in patients with NAFLD is essential for directing specialist care and improving outcomes. Although T2DM is a major risk factor for NAFLD, there are no guidelines on screening in this population.

We aimed to identify the prevalence of liver abnormalities in patients attending secondary care diabetes clinic. In addition we used serum markers to determine degree of fibrosis.

Methods All patients attending a diabetes clinic at a single centre over a 24 month period were analysed. Patients with an elevated ALT or ALP were identified. Patient demographics, clinical, imaging and laboratory characteristics were recorded. We analysed the ability to calculate Fibrosis-4 (FIB4) and NAFLD fibrosis scores (NFS) to risk stratify patients.

Results Of the 1380 patients assessed, 265 (19\%) had elevated ALT and/or ALP, after excluding patients with alternative diagnoses causing liver function derangement. The median age was 64 years, $43.8 \%$ were male, mean BMI was $31.8 \pm 6.8 \mathrm{~kg} / \mathrm{m}^{2}$.
$25.7 \%$ of patients underwent an ultrasound scan. $12 \%$ of these were suggestive of cirrhosis. Platelet count and AST were available in $85 \%$ and $25 \%$ respectively. The FIB4 or NFS was calculatable in only 65 patients (25\%). FIB4 and NFS scores identified $6 \%$ and $14 \%$ as indeterminate risk and $2 \%$ and $5 \%$ as high risk for advanced fibrosis respectively, see figure 1 . Of the indeterminate or high risk patients on either score $(n=50)$, all were older than $40,46 \%$ male and $70 \%$ had a BMI $>30 \mathrm{~kg} / \mathrm{m}^{2}$.

Conclusions We highlight minimal rates of recognition of potentially advanced liver fibrosis in a diabetic high risk population. Further education regarding risk stratification using serum markers will identify those patients who need hepatology assessment and monitoring.

\section{PTU-024 DO IRON STUDIES ON ADMISSION PREDICT OUTCOME IN PATIENTS WITH ACUTE LIVER FAILURE?}

Debbie Torland*, ${ }^{1}$ Tom Manship, ${ }^{1}$ Mhairi Donnelly, ${ }^{1}$ Maria Squires, ${ }^{2} J o a n n a$ Moore, ${ }^{3}$ Darren Craig, ${ }^{1}$ Geoff Beckett, 'Peter Hayes, ${ }^{1}$ Simon Walker, ${ }^{1}$ Ken Simpson. ${ }^{1}$ NHS Lothian, Edinburgh, UK; ${ }^{2}$ Leeds Teaching Hospitals, Leeds, UK; ${ }^{3}$ South Tees Hospital NHS Foundation Trust, , UK

\subsection{6/gutjinl-2019-BSGAbstracts.233}

Introduction Acute liver failure (ALF) has a high short term mortality. Current prognostic models lack sensitivity. This study aimed to investigate the prognostic potential of iron metabolism in ALF.

Methods 528 patients admitted to a liver transplant unit with ALF were selected. Aetiology, outcome (spontaneously survived vs. death/transplant) and hepatic encephalopathy (HE) were recorded. Patients without the relevant iron indices recorded were excluded.

Results Paracetamol overdose (POD) patients $(n=236)$ had a significantly higher transferrin if they survived $(n=168)$ compared to those that died or were transplanted $(n=68)(1.585$ $(1.363-1.918)$ vs. $1.285(1.000-1.730)(\mathrm{p}<0.001))$

In non-POD patients $(\mathrm{n}=88)$ the median transferrin was also significantly higher in those patients that survived $(n=48)$ compared to those that died or were transplanted $(n=40)$ (1.965 (1.435-2.375) vs. $1.455(1.078-1.803)(\mathrm{p}=0.001))$

In POD patients $(n=246)$ the median ferritin was significantly higher in those who died or were transplanted $(n=70)$ compared with survivors $(n=176)(36,822(13,054-64,358)$ vs.

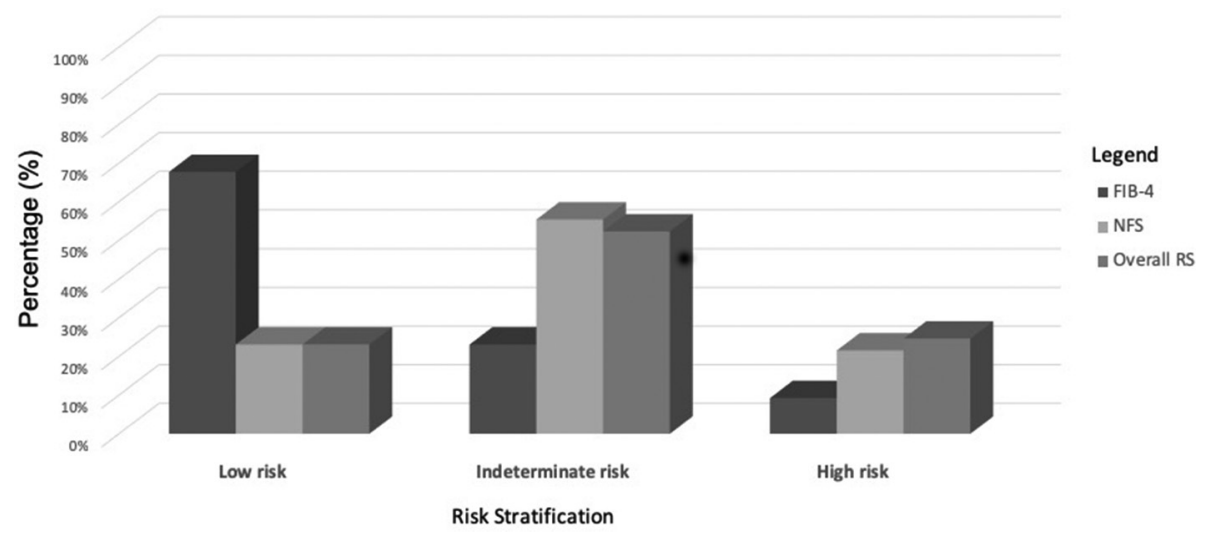

Abstract PTU-023 Figure 1 Breakdown of patients by risk stratification 